Special issue of the 2nd International Conference on Computational and Experimental Science and Engineering (ICCESEN 2015)

\title{
Collision Cascade and Spike Effects of X-ray Irradiation on Optoelectronic Devices
}

\author{
S. SAlleh ${ }^{a}$, H.F. Abdul Amir ${ }^{b}$, A. Kumar Timari ${ }^{a}$ And F. Pien Chee ${ }^{a} * *$ \\ ${ }^{a}$ Physics and Electronics Department, Faculty of Science and Natural Resources, University of Malaysia Sabah, \\ Kota Kinabalu, Sabah, Malaysia \\ ${ }^{b}$ School of Physics and Materials, Faculty of Applied Science, University Teknologi Mara, \\ Shah Alam, Selangor, Malaysia
}

\begin{abstract}
Bombardment with high energy particles and photons can cause potential hazards to the electronic systems. These effects range from degradation of performance to functional failure, which can affect the operation of a system. Such failures become increasingly likely as electronic components are getting more sophisticated, while decreasing in size and tending to a larger integration. In this paper, the effects of X-ray irradiation on a plastic encapsulated infrared light emitting diode, coupled to a plastic encapsulated silicon infrared phototransistor, with both of them being electrically isolated at $\mathrm{ON}$ and $\mathrm{OFF}$ modes, are investigated. All the devices are exposed to a total dose of up to $1000 \mathrm{mAs}$. The electrical parameters of the optoelectronic devices during the radiation exposure and at post-irradiation are compared to the pre-irradiation readings. The findings show that the highest degradation occurs at low dose of exposure; beyond $100 \mathrm{mAs}$ the relative decrease in collector current of the phototransistor is gradually reduced. The most remarkable feature found, is the operational dependence of the bias collector current, indicating a higher degradation for low bias forward current of the light emitting diode. The degradation induced at the forward current of the light emitting diode by X-rays irradiation is almost negligible whereas a decrease of the rate of change in current transfer ratio is significant during the $\mathrm{X}$-ray irradiation. The results show that there is no significant difference between current transfer ratio of ON mode and OFF mode radiation. It is observed that the operating mode of the optoelectronic devices after exposure to $1000 \mathrm{mAs}$ of X-ray irradiation contributes no major variation in the degree of damage.
\end{abstract}

DOI: 10.12693/APhysPolA.130.93

PACS/topics: 24.10.-i, 25.45.De, 25.45.Hi, 32.80.-t, 32.80.Fb, 32.80.Fb

\section{Introduction}

Ever since the beginning of nuclear detonation activities and observation of their effect on satellites, intensive study on the effects of ionizing radiation on semiconductor devices has been a concern of increasing importance. The manufacturing of higher density, with higher performance integrated circuits utilizes energetic particles or photons and this causes a significant radiation damage. A variety of radiations such as $\alpha$ particles, $\gamma$ rays, X-rays, neutrons and electrons had been used to observe the production of lattice-displacement defects in bulk crystalline semiconductor materials. The measurement techniques, for example optical absorption, electronic transport, and electronic spin resonance had been used to study the lattice defects. The secondary effects of the degradation of the lifetime of semiconductor devices, which include the radiation induced degradation and carrier removal, have also been studied.

Ionizing radiation is shown to inflict dislocation and other types of electrically active defects. Inter-diffusion of gate metal with the gallium arsenide (GaAs) results in a reduction of the active channel depth and a change in the effective channel doping [1]. Eladl [2] has examined ionizing radiation effect of neutrons on the static and

\footnotetext{
*corresponding author; e-mail: fpchee06@gmail.com
}

dynamic behavior of an optoelectronic-integrated device (OEID). Effects of different flux levels on another factor, early coefficient, can be represented through output current of OEID by the change in the base width of the transistor as a function of base-collector bias voltage, as was shown in [2].

In Wendler's [3] study on the mechanisms of damage formation in semiconductors, transfer of energy is involved between the atomic collision process (nuclear energy loss) and electronic excitations (electronic energy loss) during the implantation of energetic ions. Wendler has outlined several parameters that affect the formation of radiation damage, which depend on the ion energy, the target temperature during irradiation, the ion mass, the ion flux $j$ (number of implanted ions per unit area and time) and the ion fluence (number of ion per unit area).

Chavez, et al. have conducted ionizing irradiations at low dose rate using ${ }^{60} \mathrm{Co}$ source [4]. The results have shown that the voltage begins to decrease gradually with increasing levels of radiation, and a sudden failure occurs after a particular load level. The failure level was higher at reduced loading. In addition, parts tested under a light load have exhibited some degradation but no failures.

$\mathrm{X}$-ray synchrotron radiation, which also causes the ionizing effect, is crucial in the studies of the structural analysis of solids due to its characteristic low angular divergence and extremely high intensity. In general, detrimental effects like buildup of damage and strain 
usually occur in some processes, such as ion implantation in compound semiconductors $[5,6]$. Most of the ionizing radiation studies use $\gamma$ rays, neutrons, etc. This is because X-ray are electromagnetic radiation which is similar to $\gamma$ rays. However, $\mathrm{X}$-rays exhibit characteristics different from those of $\gamma$ rays and have an energy range $(120 \mathrm{eV}$ to $120 \mathrm{keV})$ which is far much smaller than that of $\gamma$ rays $(0.1 \mathrm{MeV}$ to $10 \mathrm{MeV})$. The study of the effects of $\mathrm{X}$-rays irradiation on electrical devices is therefore crucial, as it simulates endo-atmospheric irradiation effect, which is of lower energy.

Since most of the studies are emphasizing on shielding and material study, while most of the damages of the devices are measured only after the irradiation is completed (post-irradiation), it is through this kind of research the operating conditions and operating parameters of the system are investigated $[7,8]$. These factors might serve as inherent characteristics that play a major role in the ability of components to function properly in harsh radiation environment. This paper presents the analytical and numerical study of changes in optoelectronic devices resulting from X-ray irradiation.

\section{Radiation test procedure}

The function of optoelectronic device is based on the interaction of electronic process with light. The devices under tests (DUT) consisted of a plastic encapsulated infrared light emitting diode (IRLED) coded QEE113, coupled to a plastic encapsulated silicon infrared phototransistor coded QSE113. The QEE113 is actually a GaAs based IRLED (940 nm output) encapsulated in a wide angle medium and clear epoxy side-looker package. The QSE113 is a bipolar silicon phototransistor encapsulated in a wide angle medium with a black plastic side-looker package, transparent for infrared light. Construction of DUT is similar to the function of an optocoupler, in which the IRLED transfers the electrical signals into light waves to provide coupling while maintaining the electrical isolation between the input and output. The basic parameter for such coupled optoelectronic devices is the current transfer ratio (CTR), which gives the ratio of the phototransistor current $I_{\mathrm{C}}$ to the IRLED current $I_{\mathrm{F}}$, and thus combines quantities which depend on both the sending and the receiving component.

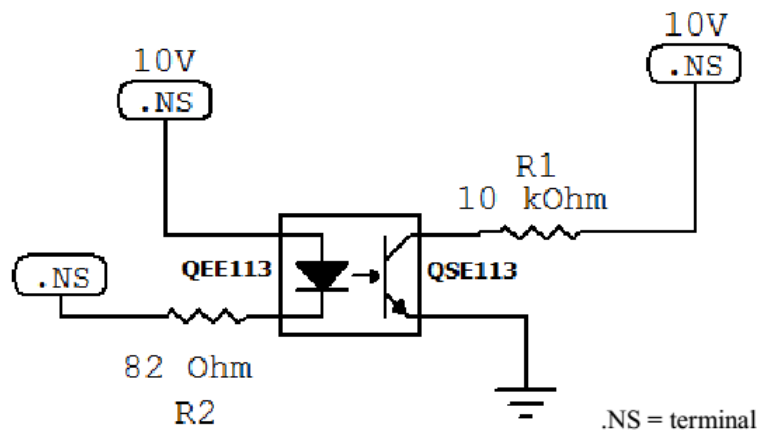

Fig. 1. Circuit of experimental setup for the optoelectronic device testing.
The radiation testing of the optoelectronic devices consists of multi-parameter tests at different exposure levels. Ambient temperature throughout the test was $25 \pm 3{ }^{\circ} \mathrm{C}$. Before the irradiation process, a control test is performed on the DUT during 72 hours, as the pre-irradiation test. Only devices which are within the specific working range and the test results of which reveal that they are working normally within the standard deviation of $2 \sigma$ (at 95 percent confidence level of normal distribution) are submitted for radiation testing. The output $I_{\mathrm{C}}$ of the phototransistor is measured for the forward current of the IRLED $I_{\mathrm{F}}$ of $25 \mathrm{~mA}, 50 \mathrm{~mA}, 75 \mathrm{~mA}$ and $100 \mathrm{~mA}$. The schematic of experimental setup of the optoelectronic device is shown in Fig. 1. The terminals for $10 \mathrm{~V}$ dc power supply and the breakdown point are marked as NS.

Every test at different bias conditions and irradiation modes was repeated 10 times using brand new devices. Two types of tests were performed in this experiment, in-flux test and post-flux test.

\subsection{In-flux test}

Measurements on the DUT were carried out during radiation exposure when both the IRLED and phototransistor were in working condition. Therefore, all changes in the parameters could be recorded and monitored directly. The input voltage of the particular device was changed from a distance of approximately $15 \mathrm{~m}$ in a control room during irradiation and the effect was observed directly using in-situ method. The schematic drawing of the test setup for in-situ testing is shown in Fig. 2.

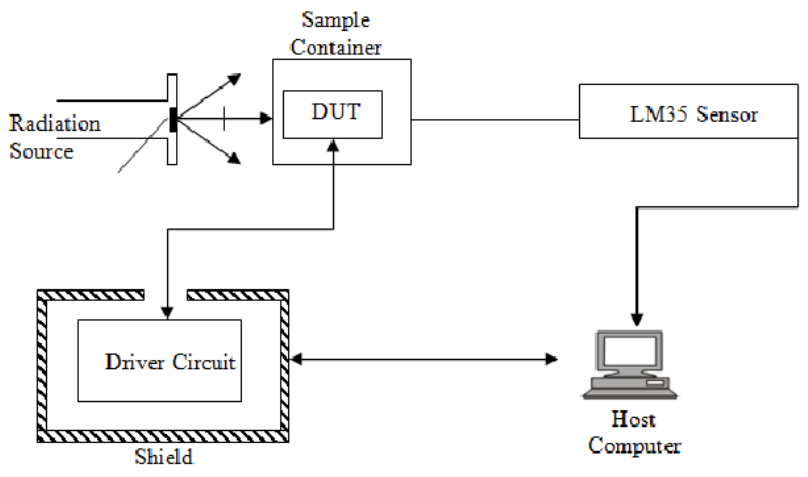

Fig. 2. Experimental arrangement for observation of changes in the DUT during irradiation.

The temperature of the tested device was monitored with an electronic thermometer based on LM35 precision centigrade temperature sensor. The information and status of the DUT were transmitted to a computer through the driver circuit based on an analogue to digital converter.

\subsection{Post-flux test}

Measurement made on DUT after the irradiation is known as post-irradiation test. It is crucial for low radiation doses or for some applications in which the DUT may reveal time-dependent effects. 
As for the X-ray source equipment, an exposure time selector and a milliampere-seconds ( $\mathrm{mAs}$ ) relay were connected to a computer. Thus the X-rays tube current could be controlled according to the selected value of the $\mathrm{mAs}$ product. The operating potential of the X-rays machine was $40 \mathrm{kV}$ and there are two exposure milliampere settings of $50 \mathrm{~mA}$ and $100 \mathrm{~mA}$. The total radiation output for an exposure period is proportional to the mAs. Therefore, the absorbed dose of the DUT could be increased by raising the mAs. Distance from the focal point of the X-rays tube to the irradiated DUT was fixed at $50 \mathrm{~cm}$. Such distance was chosen as the radiation intensity varies approximately inversely proportional to the square of the distance. The changes in the output parameter of the DUT were recorded and monitored at every increasing level of mAs. The highest mAs level that could be achieved by X-ray generator was $1000 \mathrm{mAs}$.

\section{Results and discussion}

All results including figures and tables presented in this section refer to irradiaton of the optoelectronic devices at operating conditions of ON and OFF modes using X-rays at exposure up to $1000 \mathrm{mAs}$.

The electrical parameters of optoelectronic devices for different values of applied current $I_{\mathrm{F}}$ at a step of $5 \mathrm{~mA}$, during the exposure to $\mathrm{X}$-rays in the $\mathrm{ON}$ mode were graphically depicted and tabulated. The collected current $I_{\mathrm{C}}$ of phototransistor measured before the irradiation and the parameters measured during the irradiation were compared and analysed. It was found that $I_{\mathrm{C}}$ remains constant at room temperature conditions and its readings show a minor decrease with the increase of X-ray irradiation intensity. From the trend shown in

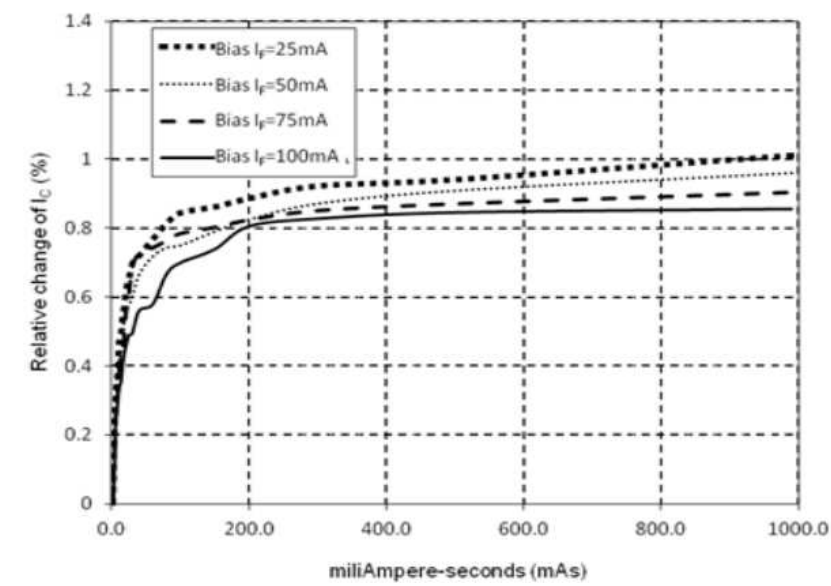

Fig. 3. Relative decrease of $I_{\mathrm{C}}$ of the phototransistor QSE113 during irradiation for different values of IRLED current $I_{\mathrm{F}}$.

Fig. 3, it is observed that the highest degradation occurred at the beginning of the exposure. After the exposure of approximately $100 \mathrm{mAs}$, the gradient of the slope is significantly depleted; this means that the relative decrease in $I_{\mathrm{C}}$ of the phototransistor is gradually reduced.
The most remarkable feature found is the operational dependence of the $I_{\mathrm{C}}$, showing a higher degradation for low bias forward current $I_{\mathrm{F}}$.

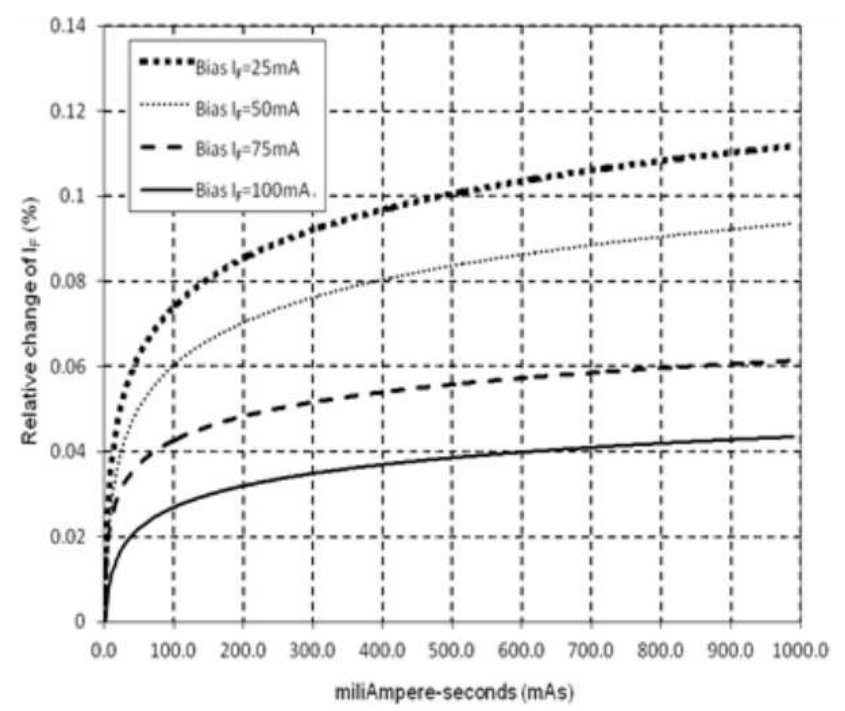

Fig. 4. Relative decrease of $I_{\mathrm{F}}$ of IRLED QEE113 during irradiation for different values of IRLED current $I_{\mathrm{F}}$.

As shown in Fig. 4, $I_{\mathrm{F}}$ shows a rapid and almost linear decrease with the dose of X-rays irradiation at the beginning of the exposure. The inclination angle, however, is reduced after the exposure of about $100 \mathrm{mAs}$. Besides, it is established that the percentage of decrease in $I_{\mathrm{F}}$ is higher at lower bias $I_{\mathrm{F}}$. However, from the obtained data, it is found that the degradation induced in the $I_{\mathrm{F}}$ of IRLED by the X-rays is almost negligible at a total exposure of $1000 \mathrm{mAs}$.

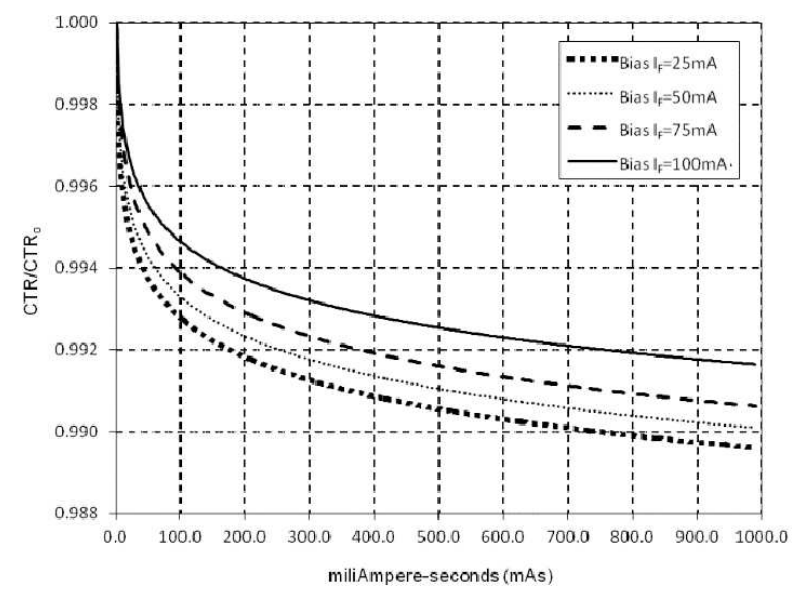

Fig. 5. $\frac{\mathrm{CTR}_{0}}{\mathrm{CTR}_{0}}$ during irradiation for different values of IRLED current $I_{\mathrm{F}}$.

The rate of change of the current transfer ratio $\frac{\mathrm{CTR}}{\mathrm{CTR}_{0}}$ of the tested optoelectronic device was monitored and recorded, where $\mathrm{CTR}_{0}$ is the initial reading of CTR. The tested degradation in the optoelectronic devices due to the exposure to $\mathrm{X}$-rays is generally causing a slight decrease of the CTR, as shown in Fig. 5. 
From Fig. 6, it is observed that CTR of the irradiated optoelectronic devices shows a noticeable increase at a slower rate after the end of the exposure. This phenomenon can be explained by the fact that radiation induces an excess of electrons in the substrate of the device, which accumulate at the interface during the exposure and lead to the decrement of the CTR. However, when the exposure is removed, these excess carriers gradually vanish via the recombination and cause a slight increase in the performance parameters.

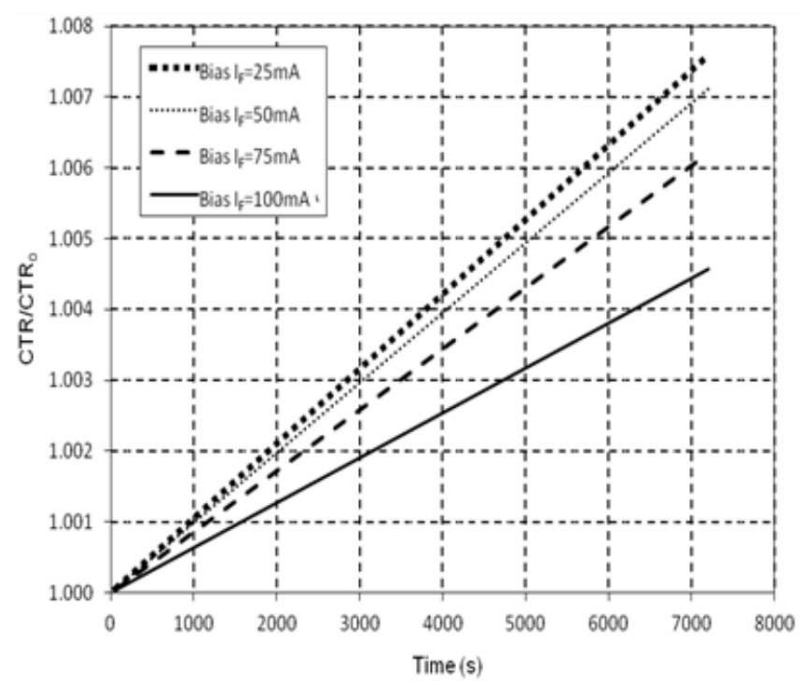

Fig. 6. $\frac{\mathrm{CTR}}{\mathrm{CTR}_{0}}$ at post-irradiation for different values of IRLED current $I_{\mathrm{F}}$.

A statistical test, t-test is applied when there is a necessity to assess the means of two group results, when two variables are statistically different from each other [9]. Thus, t-test was carried out in this study to assess the significance of the difference between the means of CTR of the ON and OFF modes after the exposure to $1000 \mathrm{mAs}$ of X-ray radiation. The significance testing result $(P>0.05)$ shows that the exposure to $\mathrm{X}$-rays at a total dose of $1000 \mathrm{mAs}$ had been causing an insignificant change in the optoelectronic system, as it can work almost ideally at post-irradiation.

When the radiation penetrates into the target layer, it undergoes a series of collisions with the atoms and electrons in the target. In these collisions, the atoms in the target layer which are displaced by the incident ions are known as primary knock-on atoms (PKAs). The PKAs can also in turn displace other atoms and end up in creating a cascade of atomic collisions [10]. This leads to the creation of vacancies and atoms in an interstitial positions and thus causes the lattice disorder in the region around the ion track. Such defects of the crystalline lattice are known as point defects. This phenomenon is illustrated in Fig. 7. Such created defects are initially free to move around at room temperature and therefore can travel long distances. These defects can either disappear from the crystal structure, through recombination at the substrate or become trapped by the impurity atoms.

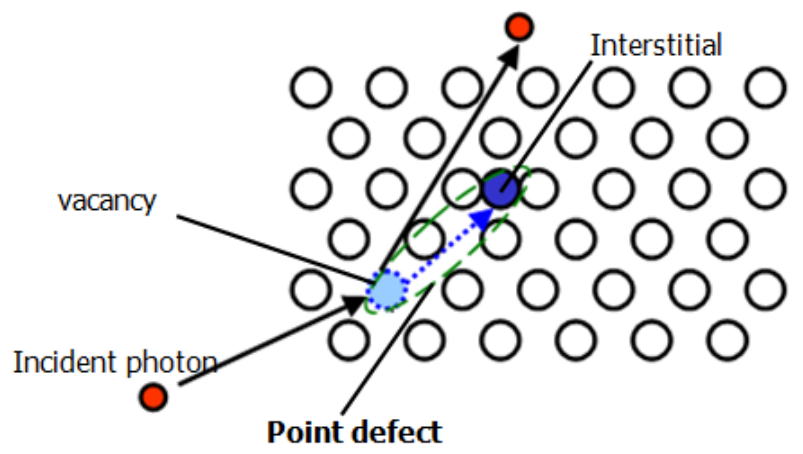

Fig. 7. Research hypothesis for the displacement damage in the crystalline structure.

The stopping of the photons, the collision sequence and subsequent ion deflection in the target materials, due to the spike effects, are stochastic processes. In these collisions, X-ray photons lose there energy at a rate $\mathrm{d} E / \mathrm{d} x$ of a few $100 \mathrm{eV}$ per nanometer, depending on their incident energy.

The X-ray photons trapped by the defects in the optical medium, give rise to more stable secondary defects and lead to formation of colour centers. The created colour centers absorb signal photons and therefore degrade the light transmission efficiency. This will result in a decrease in the phototransistor output current in current transfer applications.

The operation of these optoelectronic devices is based on the creation or annihilation of electron-hole pairs [11]. When the energetic particles, which interact with the optoelectronic device, impart energy, which is equal or larger than the bandgap energy, to a valence band electron, pair production occurs. The bandgap energy of the GaAs semiconductor material is $1.424 \mathrm{eV}$. This pair production phenomenon can raise an electron from the valence band to the conduction band, leaving a hole behind in the valence band. There is a probability that a reverse process of electron and hole recombination might occur, which is associated with the pair releasing all its excess energy after the recombination. Such recombination might be radiative or non-radiative. Radiative recombination is important for the luminescent processes. The non-radiative recombination, however, can reduce the radiative efficiency of the optoelectronic material.

\section{Conclusions}

Operating parameters can affect the level of degradation of optoelectronic devices in a given application. For the IRLED operating at a high current $\left(I_{\mathrm{F}}=100 \mathrm{~mA}\right)$, the degradation observed in both the $I_{\mathrm{C}}$ and $I_{\mathrm{F}}$ is found to be minimized significantly. This is as though in the IRLED operated at high $I_{\mathrm{F}}$ a certain amount of power that is dissipated in the resistive part of the device leads to the self-heating effect.

When significant CTR degradation is anticipated for a specific application, the effect of degradation can be 
eventually alleviated in certain cases. This can be done by decreasing the rate of CTR or by regulating the application bias condition in order to reduce the harshness of the degradation. In other words, this prediction is useful in the modeling of the devices and circuit degradation in radiation environments, without the knowledge of the exact details of the microscopic defects formed during the exposure.

\section{Acknowledgments}

The authors are thankful to Fundamental Research Grant Scheme (FRGS) 2013, Project No. FRG0318SG-1/2013, entitled "Evaluation on Diffusion of ChargeCarrier in Semiconductor and Nanostructure Devices and its Dependency on Nuclear Radiation".

\section{References}

[1] P.H. Yannakopoulos, A.P. Skountzos, M. Vesely, Microelectr. J. 39, 732 (2008).

[2] Sh.M. Eladl, Microelectr. J. 40, 193 (2009).
[3] E. Wendler, Nucl. Instrum. Methods B 267, 2680 (2009).

[4] R.M. Chavez, B.G. Rax, L.Z. Scheick, A.H. Johnston, IEEE Radiat. Effects Data Workshop 2005, 144 (2005).

[5] R. Kögler, F. Eichhorn, A. Mücklich, W. Skrorupa, C. Serre, A. Perez-Rodriguez, Vacuum 78, 177 (2005).

[6] F.A.A. Haider, F.P. Chee, Adv. Mater. Res. 173, 1 (2011).

[7] F.A.A. Haider, F.P. Chee, J. Phys.: Conf. Ser. 574, 012019 (2015).

[8] F.A.A. Haider, F.P. Chee, Adv. Mater. Res. 1108, 79 (2015).

[9] A. Celikyilmaz, I.B. Türksen, Modeling Uncertainty with Fuzzy Logic: With Recent Theory and Applications (Studies in Fuzziness and Soft Computing), Springer, New York 2009.

[10] J.H. Warner, S.R. Messenger, R.J. Walters, G.P. Summers, IEEE Trans. Nucl. Sci. 52, 2678 (2005)

[11] V.Th. Tsakiri, A.P. Skountzos, P.H. Yannakopoulos, E. Verrelli, Optoelectronic Devices and Properties, InTech, 2011. 